\title{
Changes in the prevalence of asthma in adults since 1966: the Busselton health
} study

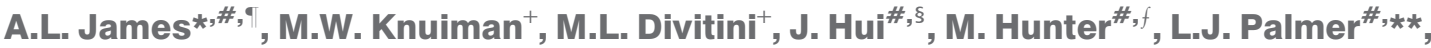 \\ G. Maier ${ }^{+}$and A.W. Musk ${ }^{\#, \varphi_{,+,}, f}$
}

ABSTRACT: Asthma prevalence has increased worldwide; although less so in developed countries recently. This study assessed changes in the prevalence of asthma and related symptoms in the Busselton community since 1966.

Cross-sectional respiratory health surveys of Busselton adults were conducted in 1966, 1969, 1972, 1975, 1981, 1990 and 2005-2007. Logistic regression models were used to estimate prevalence rates of asthma, respiratory symptoms, smoking, airway hyperresponsiveness (AHR) and atopy and to make comparisons in 2005-2007 and previous survey years. Asthma was defined as ever having doctor-diagnosed asthma (DDA).

The prevalence of DDA was around 6\% from 1966 to $1975,8 \%$ in 1981 and rose to $19 \%$ in 2005 2007. From 1981 to 2005-2007, smoking prevalence declined and obesity and atopy increased but changes in these variables explained only a small part of the increase in DDA. Wheeze and cough/ phlegm increased but AHR, breathlessness and doctor-diagnosed bronchitis remained relatively stable over the same period.

These observations indicate that the increase in DDA is partly explained by increased symptoms and atopy. The lack of changes in AHR and doctor-diagnosed bronchitis suggests that factors such as diagnostic transfer and increased awareness of asthma have also contributed to the rise in prevalence of DDA.

KEYWORDS: Asthma, epidemiology, prevalence

$\mathbf{A}$ ccording to surveys that use standardised questionnaires [1], the prevalence of asthma has been increasing worldwide over the last $30 \mathrm{yrs}$ or more, although this trend has been less evident in developing countries. The rapidity of these changes implicates environmental effects, such as exposure to tobacco smoke [2] and other pollutants [3], reduced physical activity, diet [4] and the prevalence of obesity [5, 6], and exposures to allergens [7]. The "hygiene hypothesis", which suggests that reduced earlylife exposure to infections or microbial substances increases the risk of developing allergies and asthma, has also been invoked to account for the increased prevalence of asthma especially in developed communities [1].

Since asthma is a heterogeneous disease and there is no "gold standard" for its diagnosis, a number of parameters are used to define it. In clinical practice, these include a history of variable wheezing, chest tightness and breathlessness, and demonstration of reversible airflow obstruction or airway hyperresponsiveness (AHR) to inhaled broncho-constrictors, such as methacholine, or non-isotonic stimuli [8]. Epidemiologically, a number of operational definitions of asthma have been arbitrarily used to estimate the prevalence of asthma. These have included a history of asthma ever diagnosed by a doctor and a history of wheezing (ever or within the last 12 months), sometimes in combination with tests of airway responsiveness [9]. More stringent definitions have included the presence of recent attacks of asthma, requirements for treatment with inhaled corticosteroids or unscheduled medical visits for asthma. These various definitions have led to varying estimates of the prevalence of asthma $[9,10]$.

The residents of the shire of Busselton in the south west of Western Australia have been the subject of a series of health surveys since 1966 [11, 12]. Since the initial survey, the total adult population has increased from $\sim 4,000$ to $\sim 17,000$ in 2005 . During this period, the region
AFFILIATIONS

*Dept of Pulmonary Physiology/Wes Australian Sleep Disorders Research Institute, Sir Charles Gairdner Hospital,

${ }^{\text {"Busselton Population Medical }}$ Research Foundation, Sir Charles Gairdner Hospital,

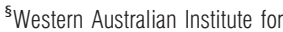
Medical Research, Sir Charles Gairdner Hospital, and

${ }^{f}$ Dept of Respiratory Medicine, Sir Charles Gairdner Hospital, Nedlands, WA,

"School of Medicine and Pharmacology, University of Western Australia,

+School of Population Health University of Western Australia, and ${ }^{* *}$ Centre for Genetic Epidemiology, University of Western Australia, Crawley, WA, Australia.

CORRESPONDENCE

A.L. James

Dept of Pulmonary Physiology/ West Australian Sleep Disorders Research Institute, Level 5, G Block,

Sir Charles Gairdner Hospital Hospital Avenue Nedlands WA 6009 Australia E-mail: Alan.James.SCGH@ health.wa.gov.au

Received:

Dec 222008

Accepted after revision: July 142009

First published online: July 302009

European Respiratory Journal Print ISSN 0903-1936 Online ISSN 1399-3003 
has remained ethnically and socio-economically stable and there has been little change in environmental influences such as industrial air pollution because viticulture, dairy farming, surfing and tourism remain the main industries. The purpose of this study was to determine the current prevalence of asthma and related symptoms in Busselton adults over the period since 1966, with special focus on the period 1981 to 2005-2007.

\section{METHODS AND MATERIALS}

\section{Study population and participant recruitment}

Cross-sectional respiratory health surveys of adults in Busselton were conducted in 1966, 1969, 1972, 1975, 1981, 1990 and more recently in 2005-2007. In each survey, the current electoral roll was used to invite adults. Registration to vote is compulsory in Australia. All adults were invited to attend surveys between 1966 and 1981 [13]; a random sample of people, aged 18-54 yrs, was invited to the 1990 survey [10]; and a random sample of all adults was invited in 2005-2007. The number of participants with adequate data was approximately 3,600 for 1966-1981 (response rates 64-91\%), 1,154 in 1990 (response rate 38\%) and 2,862 in 2005-2007 (response rate $61 \%$ ). Informed consent was obtained prior to assessments and approval for each survey was obtained from the Human Research Ethics Committee, University of Western Australia (Crawley, WA, Australia).

\section{Study protocol}

All participants completed a self-administered questionnaire that included demographic characteristics, cigarette smoking and respiratory symptoms and diagnoses; they also attended a clinic for a variety of physical measures and tests. As the wording of several questions on respiratory symptoms was slightly different prior to the 1981 survey and as AHR and atopy were not assessed prior to 1981, the main results and analyses are restricted to the surveys in 1981, 1990 and 2005-2007.

\section{Asthma, respiratory symptoms, smoking, and anthropometry measures}

Doctor-diagnosed asthma (DDA) was defined as a positive response to "Have you ever had asthma?" from 1966 to 1975 and to "Has your doctor ever told you that you had asthma or bronchial asthma?" from 1981 to 2005-2007. For the period 1981 to 2005-2007, respiratory symptoms were defined as follows: wheeze (ever) was based on response to "Have you ever had wheezing and whistling in your chest?" and wheeze (recent) was based on the follow-up question "If yes, within the last 12 months?"; shortness of breath was based on "Do you get short of breath walking with other people of your own age on level ground?" [14]; cough/phlegm was based on the report of cough or phlegm during the day or night for as many as 3 months in a year; bronchitis was based on "Has a doctor ever told you that you have bronchitis?" Several questions on smoking were included in all surveys but only current smoking is considered herein. Height and weight were measured with the participant lightly clothed and shoeless. Body mass index (BMI) was calculated and obese defined as BMI $>30 \mathrm{~kg} \cdot \mathrm{m}^{-2}$.

\section{Lung function and AHR}

Forced expiratory volume in $1 \mathrm{~s}$ (FEV1) and forced vital capacity (FVC) were measured using pneumotach spirometers
(Medical Graphics Corporation, St Paul, MN, USA) according to American Thoracic Society criteria [15] and expressed as percentages of the predicted normal values [16]. Spirometers were calibrated daily using a 3-L syringe and corrected for room temperature, humidity and barometric pressure. The dose of inhaled methacholine that provoked a $20 \%$ fall in FEV1 (PD20) was recorded using a hand-held dosimeter method [17] in 1990 or a modification [18] of it in subsequent surveys. Participants with a baseline FEV $1<60 \%$ of the predicted value received only inhaled salbutamol $(400 \mu \mathrm{g})$ and FEV1 was repeated after $15 \mathrm{~min}$. AHR was defined as a PD20 $<4 \mu \mathrm{mol}$ of methacholine or $>15 \%$ increase in FEV1 following salbutamol.

\section{Atopy}

Forearm prick skin responses to common inhaled allergens (grasses, moulds, animal proteins and house dust mite) (Hollister-Stier, Spokane, WA, USA) were assessed. Histamine $\left(10 \mathrm{mg} \cdot \mathrm{mL}^{-1}\right)$ and glycerine were used as positive and negative controls respectively. Atopy was defined as any wheal of $\geqslant 3 \mathrm{~mm}$ greater than the negative control.

\section{Statistical analysis}

Analyses were restricted to adults aged 18-79 yrs, and prevalence rates were estimated separately for males and females and for age groups 18-54 yrs and 55-79 yrs. Logistic regression models, including age and survey-year terms, were used to estimate and examine changes in the prevalence of asthma and other symptoms and conditions across the surveys after adjustment for age (in 5-yr groups). The estimated prevalence rates calculated from the fitted logistic regression models were standardised to the age distribution of the population of the study area in 2005. Repeated measures across surveys on the same individuals (who remained in the study area and attended multiple surveys) were accommodated using a generalised estimating equations regression method. Extended models, including smoking, obesity and atopy were used to examine the extent to which these variables explained the changes in prevalence of DDA across surveys. Extended models including interaction terms were used to examine whether the changes in prevalence of DDA were different by smoking status, obesity status and atopic status. The criterion of $\mathrm{p}<0.01$ was applied in the search for interactions in order to reduce the chance of false-positive findings due to multiple testing. Birth cohort effects on DDA were investigated using all surveys from 1966 to 2005-2007, and the fitted model that included age and survey year was compared with the fitted model that included birth year and survey year, as well as the examination of interactions between these time-related variables. All statistical analyses were conducted using Proc Genmod in SAS/STAT ${ }_{\circledast}$ (version 9.1.3; SAS, Cary, NC, USA).

\section{RESULTS}

Figure 1 shows the estimated prevalence of DDA in Busselton from 1966 to 2005-2007 and table 1 shows the estimated prevalence of DDA, respiratory symptoms, AHR, atopy, smoking and obesity for the 1981, 1990 and 2005-2007 surveys. The prevalence of DDA was stable from 1966 to 1975, increased slightly in 1981 and then rose sharply (and significantly) to 2005-2007. From 1981, the prevalence of DDA for both sexes was higher in the $18-54$ yrs age group than in the $55-79$ yrs 


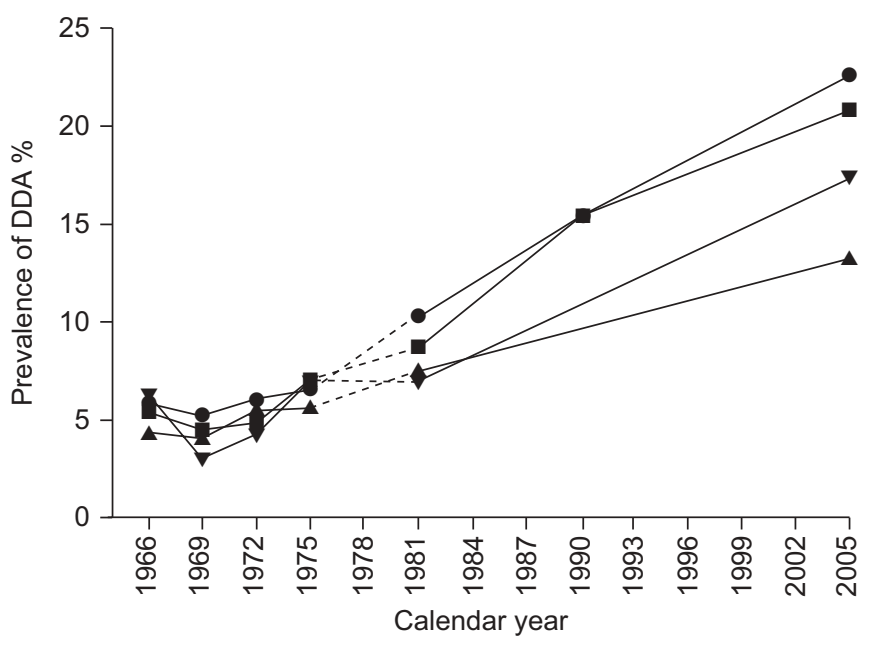

FIGURE 1. Age-adjusted prevalence of doctor-diagnosed asthma (DDA) by sex and age group in Busselton health surveys from 1966 to 2005-2007. From 1966 to 1975 , the asthma question was "Have you ever had asthma?", and from 1981 to 2005-2007 it was "Has your doctor ever told you that you have asthma?" $\mathbf{~ : ~ m a l e s ~}$ 18-54 yrs, ๑: females 18-54 yrs, $\mathbf{\Lambda}$ : males 55-79 yrs, $\mathbf{\nabla}$ : females 55-79 yrs.

age group. Wheeze (ever) and recent wheeze were common and increased from 1981 to $2005-2007$ in the 18-54 yrs age group. The prevalence of DDA plus recent wheeze increased from 1981 to 2005-2007 (especially in the 18-54 yrs group) but the prevalence of AHR plus recent wheeze was not higher in 2005-2007.

The prevalence of shortness of breath was higher in older than younger adults but changed little from 1981 to 2005-2007. The prevalence of cough/phlegm and doctor-diagnosed bronchitis were also higher in older adults but only cough/phlegm increased from 1981 to 2005-2007. Current smoking was more common in younger adults but declined from 1981 to 20052007 in both age groups and both sexes. Atopy was common, with about half of adults having a positive response to at least one antigen. The prevalence of atopy was slightly higher in younger versus older participants and, from 1981 to 2005, increased significantly in the younger adult group. The prevalence of obesity increased significantly from 1981 to 2005-2007 in all four age-sex groups.

Further modelling showed that from 1981 to 2005-2007, further adjustment for smoking, obesity and atopy (in addition to age) did not alter the changes in the prevalence of DDA across survey years. Interaction analysis did not provide any conclusive evidence that the increase in prevalence in DDA from 1981 to 2005-2007 differed by smoking status, obesity or atopic status.

Modelling using data from 1966 to 2005-2007 showed that survey year had a much stronger effect on prevalence of DDA than birth year or age. When the survey year effect was estimated after adjustment for birth year (instead of age), the survey year effect was smaller (but still highly significant): the estimated DDA odds ratio comparison of 1981 and 2005-2007 in males changed from $2.42(\mathrm{p}<0.001)$ to $1.73(\mathrm{p}<0.001)$ and in females from 2.63 ( $p<0.001$ ) to 2.11 ( $p<0.001$ ). In these models, the birth year effect suggested that adults born after $1960 \mathrm{had}$ a higher prevalence of DDA, but the data on adults born after 1960 are derived from the 1981, 1990 and 2005-2007 surveys, with two-thirds from the 2005-2007 survey, when the DDA prevalence was higher. Thus, it is unclear (and not resolvable from these data) as to whether part of the increase in prevalence of DDA from 1981 to 2005-2007 may be due to a post-1960 birth-year effect.

\section{DISCUSSION}

This analysis of data from adults in the Busselton Health Surveys from 1966 to 2005-2007 showed that the prevalence of DDA was steady from 1966 to 1975 but increased dramatically from 1981 to 2005-2007. The rising trend in DDA from 1981 to 2005-2007 was also seen for wheeze (ever), recent wheeze (younger adults only), DDA plus recent wheeze, and selfreported cough/phlegm, but not for AHR plus recent wheeze, shortness of breath, and doctor-diagnosed bronchitis (ever). Atopy increased significantly in adults $<55$ yrs from 1981 to 2005-2007. The prevalence of smoking decreased and the prevalence of obesity increased in all age groups from 1981 to 2005-2007.

Comparisons of data from different surveys may be affected by changes in the methods used to estimate prevalence. Although random samples were used in 1990 and 2005-2007 (i.e. a proportion of the total adult population) in contrast to all available adults in 1981 and earlier surveys, the population sample frame (electoral roll for Busselton shire) was the same in all surveys and the response rates and number of participants was about the same in 1981 and 2005-2007. Telephone survey of non-participants in the 2005-2007 survey showed a similar prevalence of DDA but an increased prevalence of current smoking, compared with participants. Data for non-participants for other studies, where attendance rates were higher, were not available. Although some asthma and respiratory symptom questions changed their wording slightly, they were the same from 1981 to 2005-2007 when the main increase in prevalence of DDA occurred. The change in wording of the DDA question would not explain the increase in DDA, since the narrower definition of asthma diagnosed by a doctor after 1975 would most likely lead to a reduction in prevalence. Atopy was assessed using the same classes of allergens (house dust mite, pollens/grasses, moulds, animal (cat and dog)), from the same manufacturer from 1981 to 20052007, although in 2005-2007, Dermatophagoides pteronyssinus was included in addition to Dermatophagoides farinae to assess allergy to house dust mite, while grass mix \#7 and rye grass were used to assess pollen allergy, excluding orchard grass and timothy grass that had been used in the 1981 and 1990 surveys, respectively. Atopy in all surveys was defined as a positive response to any allergen. Tests of airway responsiveness were undertaken using histamine in 1981 and 1990 and methacholine in 2005-2007. These agents have shown equivalence in testing in previous laboratory and epidemiological studies [18]. AHR was always defined as a PD20 $<4 \mu \mathrm{mol}$ of methacholine or histamine, or a $15 \%$ increase in FEV1 following salbutamol in subjects with an initial FEV1 that was $<60 \%$ of the predicted value.

The present study shows that the previously reported increase in DDA from 1981 to 1990 in Busselton residents aged $<55 \mathrm{yrs}$ [9] has continued through to 2005-2007 and that a similar 


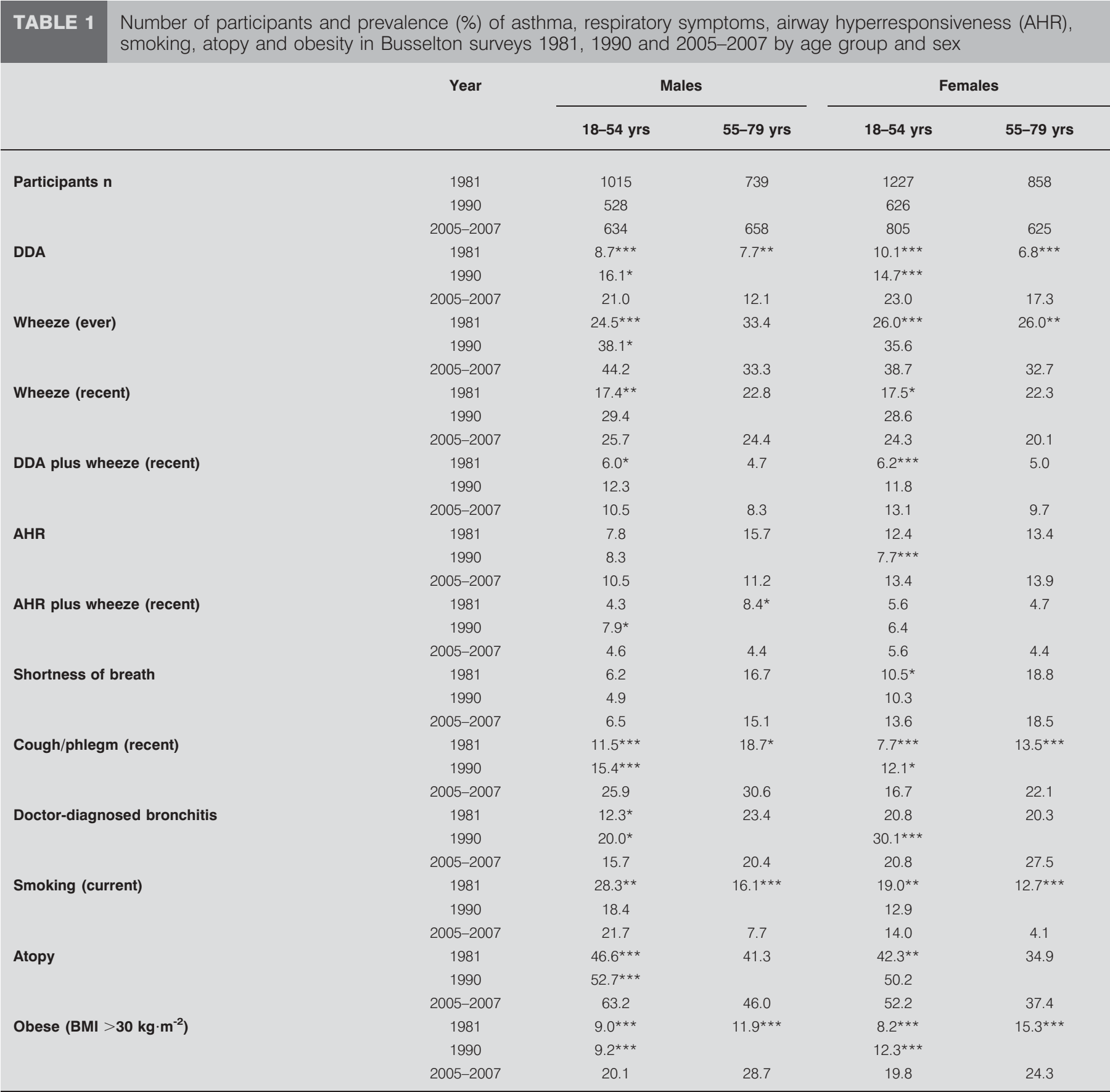

Data are presented as \%, unless otherwise stated. DDA: doctor-diagnosed asthma; BMI: body mass index. *: $p<0.05$ compared with 2005-2007; **: p<0.01 compared with 2005-2007; ***: $\mathrm{p}<0.001$ compared with 2005-2007.

increase has occurred from 1981 to 2005-2007 in participants aged 55-79 yrs. There was no clear trend from 1966 to 1975 with a prevalence of DDA around $5 \%$. Similar trends for adults were reported by WILSON et al. [19] from South Australia. They examined data from 1990 to 2003 and defined asthma as answering yes to "Has your doctor ever told you that you have asthma?" plus "Do you still have asthma?" There have also been similar increases in the prevalence of asthma in adults in studies outside Australia [20-22]. The studies of LINNEBERG et al. [20] and HANSEN et al. [22] were confined to adults aged $<41$ and 35 yrs, respectively. In the UK, as elsewhere, there are fewer data available for adults than for children but analyses of a number of studies have shown that there has generally been an increase in DDA in adults [23]. Longitudinal studies of asthma have shown that the use of questionnaire responses related to DDA in adults is affected by recall bias [24]. However, the consistent trends of symptoms observed in this study, the comparability of other studies and the lack of a gold 
standard for asthma diagnosis suggest that we will continue to rely on such questionnaire data to some extent to estimate trends in asthma.

The recorded increase in DDA and respiratory symptoms, despite a steady decline in smoking, may be due to an increase in airway inflammation and/or remodelling that is associated with asthma. Consistent with this possibility are data that show a small increase in the per cent of atopic males and females. This has been commented on elsewhere [23]. A number of other mechanisms have been suggested to contribute to a rise in asthma symptoms, including weight gain and lifestyle factors, which result in reduced cardiovascular fitness and breathlessness [5, 8, 25, 26]. However, these possibilities are not supported by the lack of change from 1981 to 2005-2007 in shortness of breath or AHR. In addition, we found that adjustment for smoking, obesity and atopy did not alter the trends in DDA. It is also possible that the increase in DDA arises from the diagnostic transfer or mislabelling of respiratory symptoms, which are increasing in prevalence, like asthma. There was an increase in wheeze observed from 1981 to 2005-2007 in all age groups, except older males, and there was an increase in the prevalence of self-reported cough or phlegm despite the marked reduction in the per cent of current smokers in the population. It is possible that these symptoms have more frequently become labelled as due to asthma since, over the last $40 \mathrm{yrs}$, there have been efforts to increase awareness of asthma in the medical community and a proliferation of easily administered and safe medications available for the treatment of asthma. Diagnostic transfer may also contribute to an apparent increase in asthma prevalence. This has previously been observed in a Western Australian paediatric population [27], where the increase in asthma diagnosis was mirrored by a decrease in the diagnosis of other respiratory conditions. The absence of change in the prevalence of doctor-diagnosed bronchitis from 1981 to 20052007, while reported current cough/phlegm and DDA increased, fits with this pattern. The propensity to diagnose asthma has increased since the early 1980 s, prior to which, a perceived reluctance to diagnose this chronic condition was observed [28, 29]. Concerns regarding untreated asthma, publication and awareness of risk factors for fatal asthma, and positive incentives from government for the diagnosis and treatment of asthma in Australia such as the "Asthma 3+ Visit Plan" and the "Asthma Cycle of Care" [30] may all have contributed to this scenario. Our age-period cohort modelling was not conclusive because most of the data on adult participants born after 1960 come from the 2005-2007 survey but nevertheless indicates that only a small part of the increase in prevalence in DDA since 1981 might be due to a post-1960 birth cohort effect.

The Australian Centre for Asthma Monitoring (ACAM) [10] reported that, based on National Health Surveys in 2001 and 2004-2005, the prevalence of reported DDA has remained stable at around $20 \%$ for males and females with a trend to reduced prevalence in older versus younger adults. These levels of prevalence are similar to the data from the present Busselton study. ACAM [10] also reported that the prevalence of current asthma, defined as a positive response to the questions "Has your doctor ever told you that you have asthma?" and "Do you still have asthma?", was 9-12\% for adults aged $<54 \mathrm{yrs}$ and $7-11 \%$ for adults aged $>54 \mathrm{yrs}$, similar to values from the present study. The increase in the prevalence of asthma (DDA and "yes, I still have asthma") reported in South Australia from 1990 to 2003 [19] was greatest in older adults and was associated with increased body weight. These findings are in contrast to our observations for DDA where the increase since 1981 was larger in younger adults and the change in prevalence was independent of current body weight.

There have been a number of international studies that have measured changes in the prevalence of asthma in adults in different parts of the world. Using varying definitions, studies have shown that, as we observed, the prevalence of DDA has increased more than have symptoms [31, 32]. Other studies have not found significant changes in asthma prevalence in adults [33, 34], although the latter study [34] was confined to adults aged $>70$ yrs.

In conclusion, the present study has demonstrated a marked increase in the prevalence of DDA in adults from 1981 to 2005 2007 in the Busselton community. The absence of a change in $\mathrm{AHR}$, the increase in current cough and phlegm and the lack of an increase in diagnosed bronchitis suggest that changes in diagnostic labels may contribute to the apparent increase in "asthma". This needs to be considered in future studies of asthma prevalence and adds to phenotypic uncertainty in defining asthma [35].

\section{SUPPORT STATEMENT}

The study was supported by the National Health and Medical Research Council Australia ID 353532.

\section{STATEMENT OF INTEREST}

None declared.

\section{ACKNOWLEDGEMENTS}

The authors would like to thank H. Bartholomew (School of Population Health, University of Western Australia, WA, Australia) for database design and programming support during the survey; P. Maxwell (West Australian Sleep Disorders Research Institute, Dept of Pulmonary Physiology, Sir Charles Gairdner Hospital, Nedlands, WA, Australia) for logistical support, training and preparation of the manuscript; D. Whittall (Busselton Population Medical Research Foundation, Sir Charles Gairdner Hospital, Nedlands, WA, Australia) for logistical support; and the numerous Busselton community volunteers who assisted with data collection and the study participants from the Shire of Busselton.

\section{REFERENCES}

1 Eder W, Ege MJ, von Mutius E. The asthma epidemic. N Engl J Med 2006; 355: 2226-2235.

2 Strachan DP, Butland BK, Anderson HR. Incidence and prognosis of asthma and wheezing illness from early childhood to age 33 in a national British cohort. BMJ 1996; 312: 1195-1199.

3 Tatum AJ, Shapiro GG. The effects of outdoor air pollution and tobacco smoke on asthma. Immunol Allergy Clin North Am 2005; 25: 15-30.

4 Ellwood P, Asher MI, Bjorksten B, et al. Diet and asthma, allergic rhinoconjunctivitis and atopic eczema symptom prevalence: an ecological analysis of the International Study of Asthma and Allergies in Childhood (ISAAC) data. Eur Respir J 2001; 17: 436-443. 
5 Chinn S. Concurrent trends in asthma and obesity. Thorax 2005; 60: 3-4.

6 Schaub B, von Mutius E. Obesity and asthma, what are the links? Curr Opin Allergy Clin Immunol 2005; 5: 185-193.

7 Illi S, von Mutius E, Lau S, et al. Perennial allergen sensitisation early in life and chronic asthma in children: a birth cohort study. Lancet 2006; 368: 763-770.

8 Sterk PJ, Fabbri LM, Quanjer PH, et al. Airway responsiveness. Standardized challenge testing with pharmacological, physical and sensitizing stimuli in adults. Report Working Party Standardization of Lung Function Tests, European Community for Steel and Coal. Official statement of the European Respiratory Society. Eur Respir J 1993; 6: Suppl. 16, 53-83.

9 Peat JK, Haby M, Spijker J, et al. Prevalence of asthma in adults in Busselton, Western Australia. BMJ 1992; 305: 1326-1329.

10 Australian Centre for Asthma Monitoring. Asthma in Australia. Canberra, Australian Inititute of Health and Welfare, 2008.

11 Cullen KJ. Chronic bronchitis and the Australian environment. Med J Aust 1972; 1: 249-253.

12 James AL, Palmer LJ, Kicic E, et al. Decline in lung function in the Busselton health study: the effects of asthma and cigarette smoking. Am J Respir Crit Care Med 2005; 171: 109-114.

13 Cullen KJ, Stenhouse NS, Welborn TA, et al. Chronic respiratory disease in a rural community. Lancet 1968; 2: 657-660.

14 Medical research council on the aetiology of chronic bronchitis. Standardised questionnaire on respiratory symptoms. $\mathrm{Br} \mathrm{Med} J$ 1960; 2: 1665.

15 Miller MR, Hankinson J, Brusasco V, et al. Standardisation of spirometry. Eur Respir J 2005; 26: 319-338.

16 Hankinson JL, Odencrantz JR, Fedan KB. Spirometric reference values from a sample of the general US population. Am J Respir Crit Care Med 1999; 159: 179-187.

17 Yan K, Salome C, Woolcock AJ. Rapid method for measurement of bronchial responsiveness. Thorax 1983; 38: 760-765.

18 James A, Ryan G. Testing airway responsiveness using inhaled methacholine or histamine. Respirology 1997; 2: 97-105.

19 Wilson DH, Adams RJ, Tucker G, et al. Trends in asthma prevalence and population changes in South Australia, 19902003. Med J Aust 2006; 184: 226-229.

20 Linneberg A, Nielsen NH, Madsen F, et al. Secular trends of allergic asthma in Danish adults. The Copenhagen allergy study. Respir Med 2001; 95: 258-264.
21 Brogger J, Bakke P, Eide GE, et al. Long-term changes in adult asthma prevalence. Eur Respir J 2003; 21: 468-472.

22 Hansen EF, Rappeport $Y$, Vestbo J, et al. Increase in prevalence and severity of asthma in young adults in Copenhagen. Thorax 2000; 55: 833-836.

23 Anderson HR, Gupta R, Strachan DP, et al. 50 years of asthma: UK trends from 1955 to 2004. Thorax 2007; 62: 85-90.

24 Burgess JA, Walters EH, Byrnes GB, et al. Who remembers whether they had asthma as children? J Asthma 2006; 43: 727-730.

25 Appleton SL, Adams RJ, Wilson DH, et al. Central obesity is associated with nonatopic but not atopic asthma in a representative population sample. J Allergy Clin Immunol 2006; 118 1284-1291.

26 Beuther DA, Sutherland ER. Overweight, obesity, and incident asthma: a meta-analysis of prospective epidemiologic studies. Am J Respir Crit Care Med 2007; 175: 661-666.

27 Carman PG, Landau LI. Increased paediatric admissions with asthma in Western Australia - a problem of diagnosis? Med J Aust 1990; 152: 23-26.

28 Williams H, McNicol KN. Prevalence, natural history, and relationship of wheezy bronchitis and asthma in children. An epidemiological study. Br Med J 1969; 4: 321-325.

29 Speight AN, Lee DA, Hey EN. Underdiagnosis and undertreatment of asthma in childhood. Br Med J (Clin Res Ed) 1983; 286: $1253-1256$

30 National Asthma Council Australia. Asthma Cycle of Care (formerly the Asthma 3+ Visit Plan). www.nationalasthma.org. $\mathrm{au} / \mathrm{html} / \mathrm{management} / \mathrm{acc} /$ index.asp. Date last accessed December 1, 2008.

31 Chinn S, Jarvis D, Burney P, et al. Increase in diagnosed asthma but not in symptoms in the European Community Respiratory Health Survey. Thorax 2004; 59: 646-651.

32 Verlato G, Corsico A, Villani S, et al. Is the prevalence of adult asthma and allergic rhinitis still increasing? Results of an Italian study. J Allergy Clin Immunol 2003; 111: 1232-1238.

33 Barraclough R, Devereux G, Hendrick DJ, et al. Apparent but not real increase in asthma prevalence during the 1990s. Eur Respir 2002; 20: 826-833.

34 Eagan TM, Bakke PS, Eide GE, et al. Incidence of asthma and respiratory symptoms by sex, age and smoking in a community study. Eur Respir J 2002; 19: 599-605.

35 Asthma: still more questions than answers. Lancet 2008; 372: 1009 